\title{
Ameloplastia positiva en relación céntrica como método auxiliar durante la finalización en el tratamiento de ortodoncia en un paciente bruxista.
}

\author{
Positive ameloplasty in centric relationship as an auxiliary method during \\ finalization in the orthodontic treatment in a bruxist patient.
}

\author{
Francisco Espinosa Albo,* Yuridia Michelle Avalos Equihua,* \\ Pablo Cesar Martínez Cardona,* Melissa Hernández Bozada,* \\ José Sayavedra Jaimes, ${ }^{\ddagger}$ Juan Saul Varela Flores, ${ }^{\ddagger}$ Salvador Antonio Ferrer Tamburini, ${ }^{\ddagger}$ \\ Claudia Salinas Escoboza, ${ }^{\ddagger}$ Oscar Vargas Yáñez ${ }^{\ddagger}$
}

\section{RESUMEN}

A continuación se presenta un caso de un paciente de 12 años de edad, con antecedente de bruxismo, con biotipo mesofacial, tercio inferior dolicofacial, hipertonicidad muscular en maseteros, cierre labial forzado con incompetencia labial de 4 milímetros con dolor en sinoviales anteriores inferiores. Los objetivos de tratamiento consistieron en lograr relajación muscular y promover un posicionamiento condilar ortopédicamente funcional y estable, realizar control vertical para disminuir gap interlabial, alinear líneas medias, mantener clase I molar bilateral, clase I canina bilateral y clase I incisiva, crear overjet y overbite adecuados, con recuperación estética y función del segmento anterior. Debido a eventos adversos durante la etapa de cierre de espacios, la clase molar y canina I no se logró, pero se aseguró mediante ameloplastias positivas la función y estética adecuadas para tener un tratamiento de ortodoncia estable a largo plazo.

Palabras clave: Bioestética, tratamiento de ortodoncia, ameloplastia, bruxismo.
ABSTRACT

The following is a case of a 12-year-old patient, with a history of bruxism, with a mesofacial biotype, lower third of the facial area, muscular hypertonicity in the masseters, a forced labial closure with a 4-millimeter labial incompetence with pain in inferior anterior synoviums. Treatment objectives consisted of achieving muscle relaxation and promoting orthopedically functional and stable condylar positioning, perform vertical control to decrease interlabial gap, align midlines, maintain bilateral molar class I, bilateral canine class I and incisive class I, create adequate overjet and overbite, with aesthetic recovery and function of the anterior segment. Due to adverse events during the closing phase of spaces, the molar and canine I class was not achieved, but positive function and aesthetics were ensured by positive ameloplasties in order to have a long-term stable orthodontic treatment.

Keywords: Bioesthetics, orthodontic treatment, ameloplasty, bruxism.

\section{INTRODUCCIÓN}

$\mathrm{E}$ I bruxismo es una parafunción mandibular persistente (hábito) que consiste en el apriete y rechinamiento

\footnotetext{
* Residente.

₹ Tutor.

Maestría en Ortodoncia, Universidad De La Salle Bajío. León, Guanajuato.

Recibido:27 Noviembre 2018.

Aceptado para publicación: 12 Marzo 2020.
}

dentario. Algunos autores creen que existen dos causas separadas de bruxismo: origen psicogénico y las desarmonías oclusales. El bruxismo se complica por desarmonías oclusales y estrés emocional como el común denominador. Otra causa puede ser la falta de una guía anterior correcta que produce deficiente actividad que puede ocasionar trauma en los dientes anteriores. ${ }^{1,2}$

Según Dawson (1974) en su experiencia clínica, el bruxismo puede detenerse gracias a la completa eliminación de todas las interferencias oclusales, estas interferencias pueden ser terceros molares retenidos hasta la edad 
adulta. Los pacientes comienzan a tener un patrón de masticación más anterior para evitar la oclusión posterior, lo que ocasiona la pérdida de la guía anterior. Dar a los pacientes guía anterior ha tenido excelentes resultados, reduciendo el hábito al mínimo. Esto nos lleva a la conclusión de que la guía anterior tienen una gran influencia con el sistema nervioso central.

Bioestética significa dar a las cosas sus formas y funciones naturales. Mediante el tratamiento bioestético podemos corregir la oclusión, cuando los resultados del tratamiento de ortodoncia fueron limitados. ${ }^{2}$

\section{PRESENTACIÓN DE CASO CLÍNICO}

Paciente femenino de 12 años de edad acudió a la Clínica de la Maestría en Ortodoncia de la Universidad De La Salle Bajío con motivo de consulta «aprieto mucho la mandíbula y no quiero que se me enchuequen los dientes», con antecedente de bruxismo (Figura 1).

En el examen clínico extraoral se determinaron características específicas, se observó un paciente de biotipo mesofacial, tercio inferior dolicofacial, hipertonicidad muscular en maseteros, cierre labial forzado con incompetencia labial de 4 milímetros, elevación asimétrica de labio superior, sonrisa invertida, canting oclusal de lado izquierdo y desviación de línea media superior 1 milímetro hacia lado derecho respecto a la línea media facial (Figura 2).

En el análisis clínico intraoral se observó dentición permanente, desgaste en dientes anteriores, clase I molar bilateral, clase I canina bilateral, clase I incisiva con tendencia a mordida abierta, overjet aumentado con 2.5 $\mathrm{mm}$, curva de Spee aumentada $2.5 \mathrm{~mm}$, discrepancia óseo-dentaria negativa en ambas arcadas.

Al examinar la oclusión dinámica, la paciente presentó ausencia de guías funcionales con interferencias generalizadas, protrusión con rango de avance normal, apertura aumentada y lateralidades con desplazamiento disminuido.

Para diagnosticar el estado de salud de la articulación temporomandibular se utilizó el mapa del dolor del Dr. Mariano Rocabado, revelando puntos dolorosos en sinoviales anteriores inferiores.

El montaje de modelos de estudio en tentativa de relación céntrica evidenció el punto de contacto prematuro en cúspide palatina de primer premolar superior izquierdo y vertiente vestibular de primer premolar inferior izquierdo. El indicador de posición condilar utilizado por el articulador mostró una retrusión y detrusión condílea de $1 \mathrm{~mm}$ del lado derecho, y detrusión condílea de 1 $\mathrm{mm}$ del lado izquierdo (Figura 3).

\section{Objetivos de tratamiento}

Con base en las características determinadas en el análisis diagnóstico, se trazaron objetivos específicos, los cuales consistieron en lograr relajación muscular y promover un posicionamiento condilar ortopédicamente funcional y estable, realizar control vertical para disminuir gap interlabial, alinear líneas medias, mantener clase I molar bilateral, clase I canina bilateral y clase I incisiva, crear overjet y overbite adecuados, nivelar plano oclusal, eliminar discrepancia óseo-dentaria negativa de ambas arcadas, crear guías funcionales sin interferencias con rangos de excursivos mandibulares normales en ausencia de sintomatología articular y conseguir una oclusión orgánica.

\section{Plan de tratamiento}

Una vez determinados los objetivos de tratamiento, se propuso una ruta terapéutica que consistió principalmente en colocar un plano desprogramador neuromuscular superior confeccionado en relación céntrica con puntos de contacto simultáneos y guías funcionales.

Después de la terapia de disfunción articular, se determinó realizar una mecánica ortodóncica que consistió en la extracción de segundos premolares superiores e inferiores, alineación y nivelación, cierre de espacios utilizando mecánica de anclaje moderado para arcada superior y mínimo anclaje para arcada inferior, coordinación de arcadas, ajuste oclusal y finalización del caso.

\section{Progreso del tratamiento}

Se comenzó utilizando un plano desprogramador neuromuscular superior, el cual fue ajustado semanalmente con puntos de contacto simultáneos de la misma intensidad y guías funcionales. Se dio indicación de uso 24 horas sólo retirando el guarda oclusal para realizar el aseo bucal (Figura 4). Tras cuatro meses de tratamiento, se realizó revaloración del caso que mostró asentamiento condilar en relación céntrica, eliminando sintomatología articular y se obtuvieron guías y movimientos mandibulares cómodos y fluidos (Figura 5).

Se inició con el tratamiento ortodóncico para el cual se utilizaron brackets Synthesis de la casa comercial ORMCO con prescripción Roth Slot 0.022 (Figura 6).

Se colocó la aparatología fija en ambas arcadas, iniciando con arcos de nivelación 0.014 CuNiTi, 0.016 CuNiTi, 0.018 CuNiTi, $0.016 \times 0.016$ CuNiTi, 0.020 $\times 0.020 \mathrm{CuNiTi}, 0.019 \times 0.025 \mathrm{CuNiTi}$, al mismo tiempo se colocaron topes posteriores céntricos para 

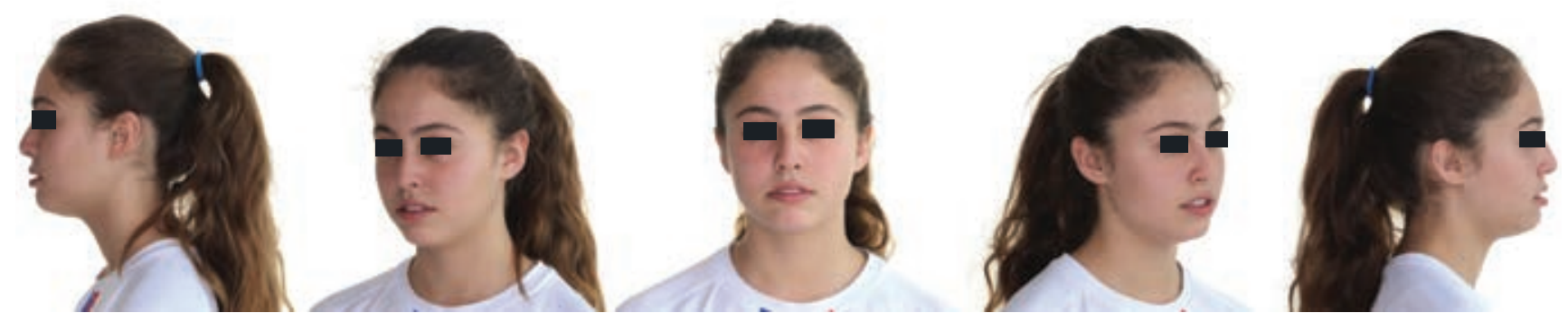

Figura 1: Facial inicial.

Figura 2:

Fotografías iniciales.
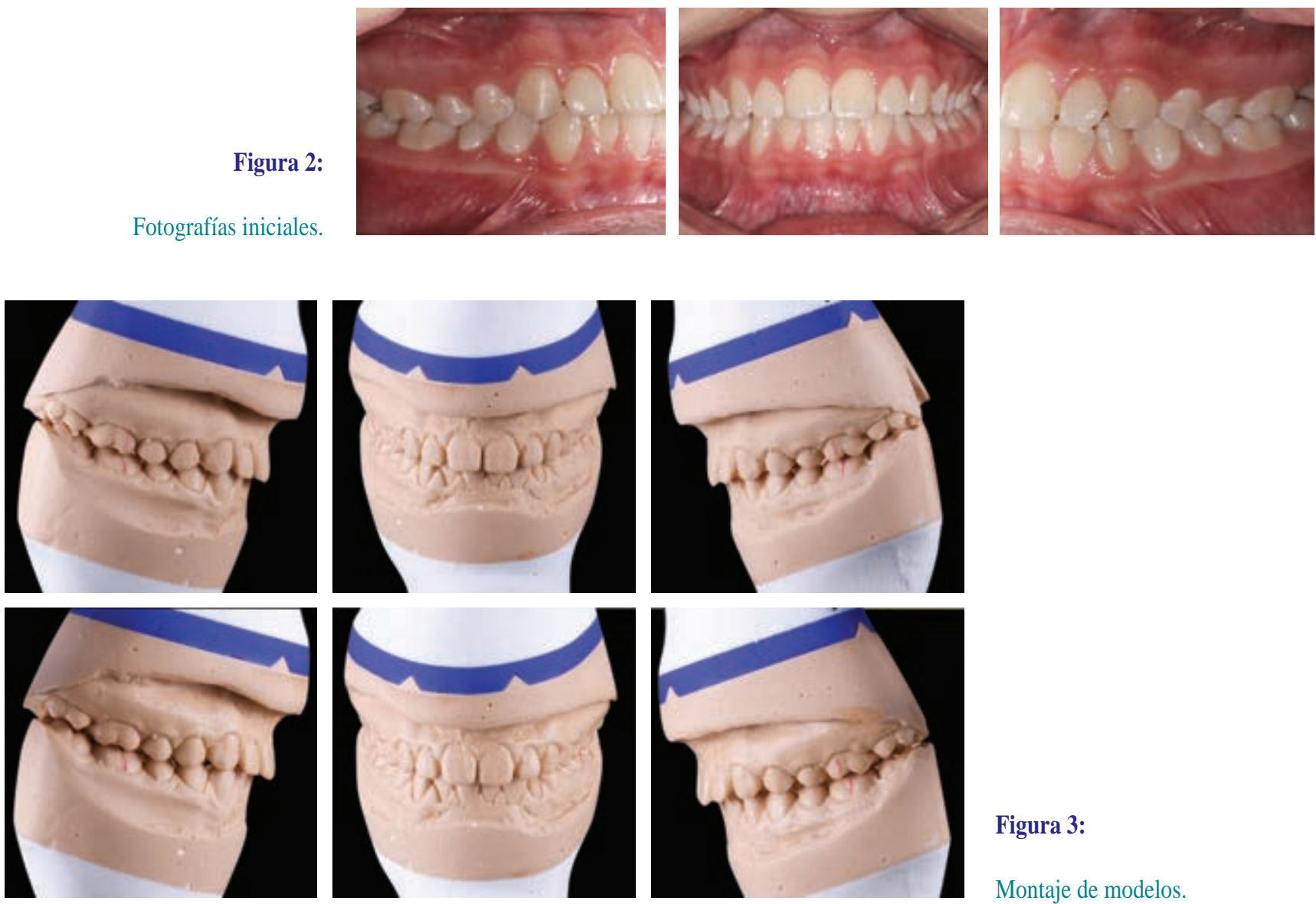

Figura 3:

Montaje de modelos.

Figura 4:

Fotografías iniciales con guarda. oclusal.
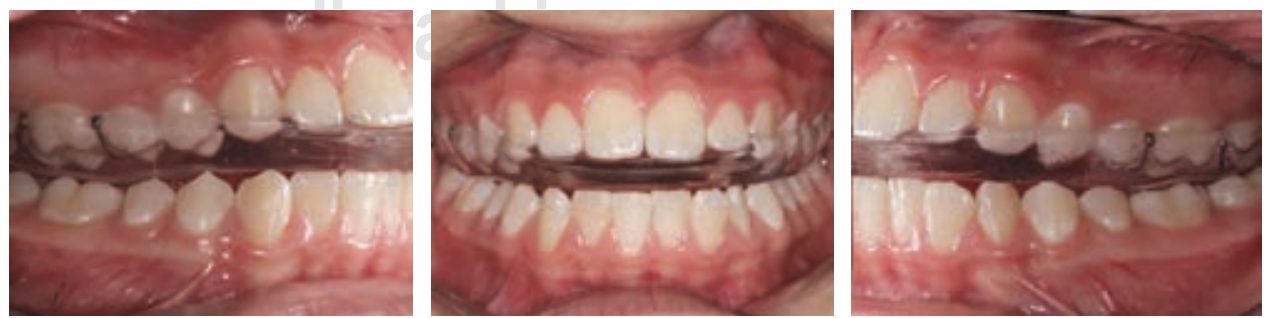

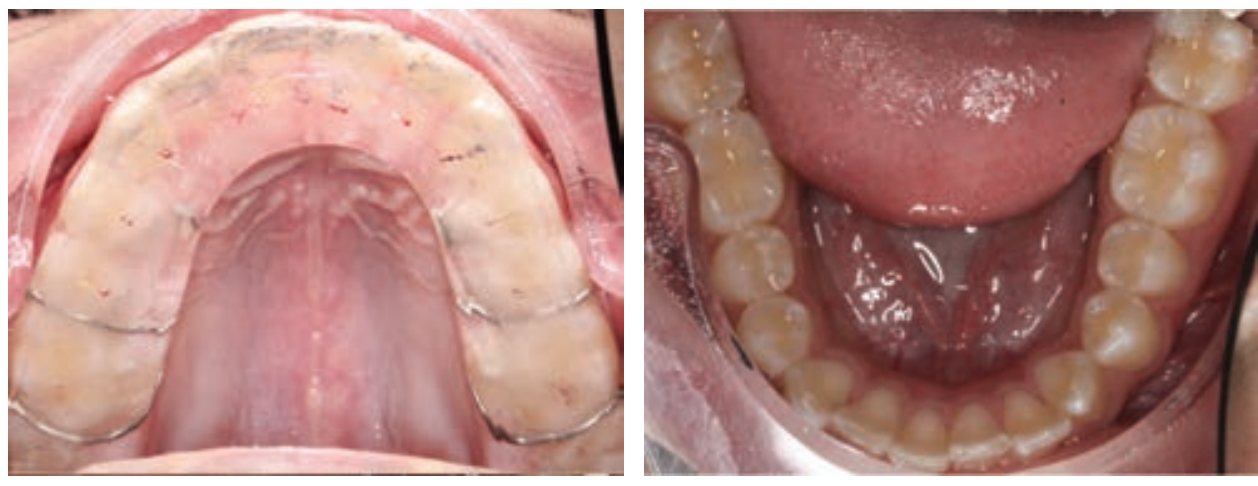

Figura 5:

Vista oclusal superior e inferior.
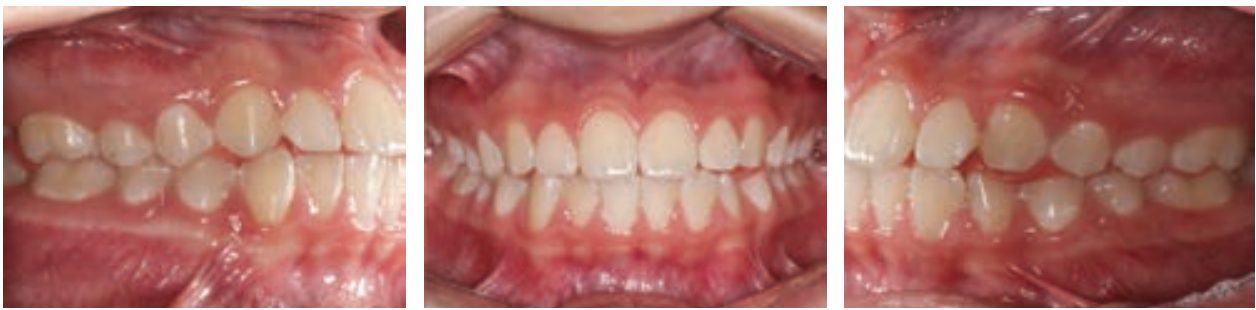

Figura 6:
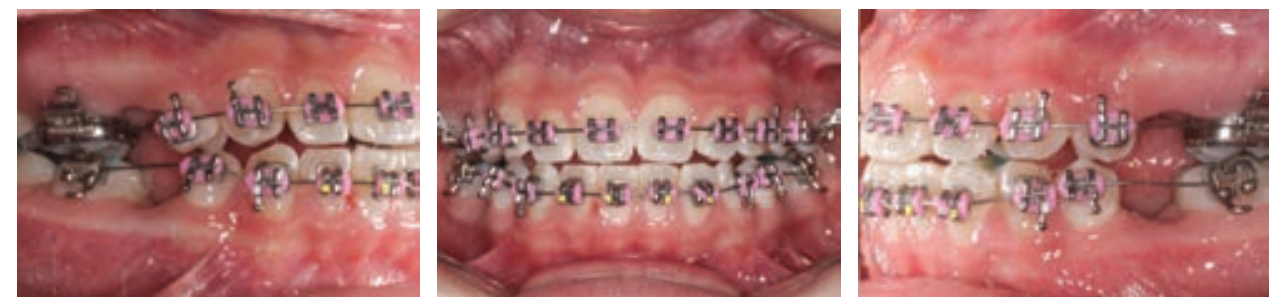

Alta después del tratamiento.
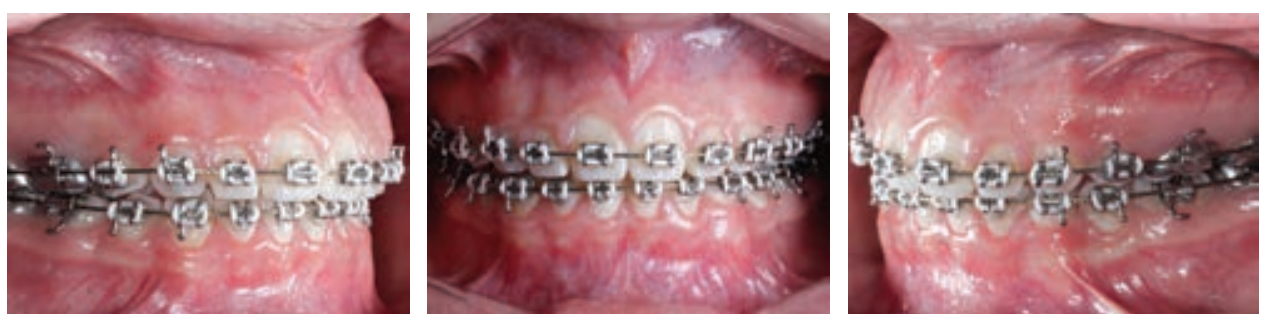

Figura 7:

Tratamiento de ortodoncia inicial.

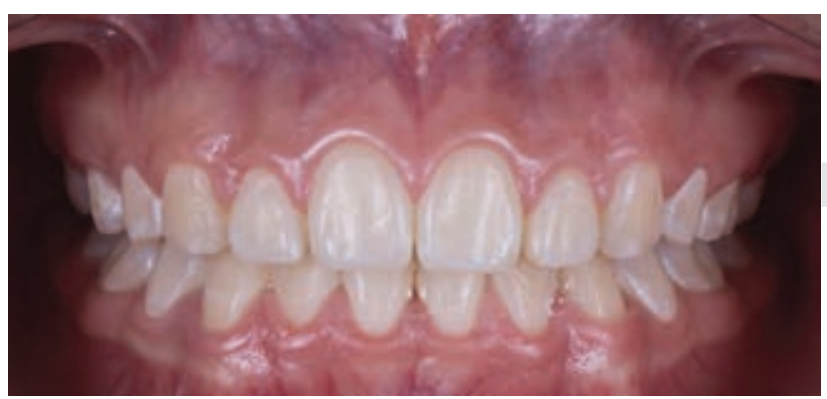

Figura 8: Avance del caso. realizar control vertical y disminuir incompetencia labial existente.

Una vez lograda la alineación y nivelación, se colocaron arcos $0.019 \times 0.025$ SS en ambas arcadas para iniciar la etapa de cierre de espacios con mecánica de mínimo anclaje en arcada inferior, ferulizando el segmento anterior con ligadura metálica y arco lingual elaborado con alambre de acero de calibre 0.036 y colocando stops de resina en distal de caninos inferiores, se redondearon los segmentos posteriores del arco y se colocó cadena elástica de caninos a molares inferiores para favorecer el cierre de 

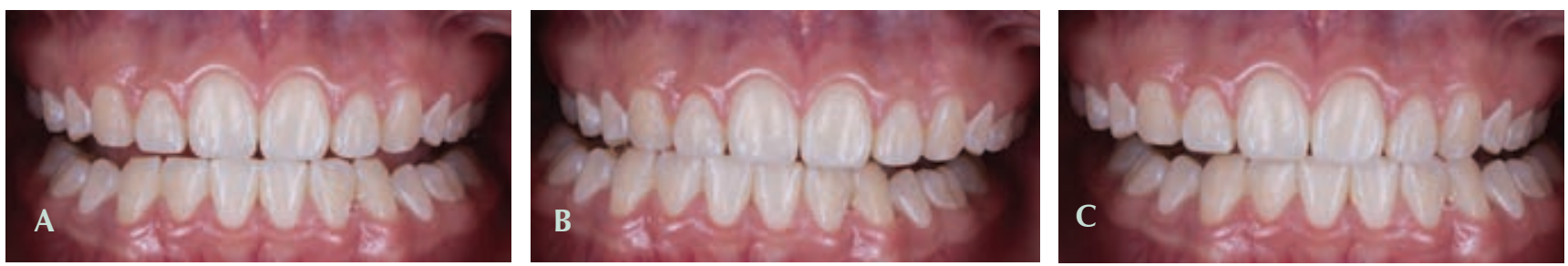

Figura 9: A) Alta con guía incisiva. B) Vista de lateralidad derecha. C) Vista de lateralidad izquierda.

espacios. En arcada superior, el espacio fue consumido durante la etapa de alineación y nivelación, por lo que se colocaron una cadenas elásticas en la totalidad de la arcada para consolidar el cierre de espacios (Figura 7).

Como coadyuvante a la mecánica de cierre de espacios en la arcada inferior, se utilizaron elásticos de $1 / 8$ pesados de clase II de caninos superiores a primeros molares inferiores (Figura 8).

Durante esta etapa hubo desalojo del arco lingual en varias ocasiones, por lo que el anclaje se vio afectado al mismo tiempo que la paciente tuvo periodos largos de inasistencia a sus citas de control, lo cual contribuyó a la profundización de la curva de Spee y a la retroinclinación de los incisivos inferiores.

Debido a múltiples eventos adversos relacionados con la cooperación del paciente, se decidió continuar con la etapa de finalización del caso en la que se colocaron arcos trenzados $0.019 \times 0.025$ con módulos elásticos individuales para el asentamiento de la oclusión (Figura 9).

Tras 30 meses de tratamiento, se retiró aparatología fija y se observó desgaste en segmentos anteriores, por lo que se realizó montaje en relación céntrica para la elaboración de un encerado con la finalidad de crear una llave de transferencia para realizar ameloplastias positivas en ambos segmentos con el objetivo de mejorar las guías funcionales, estética y una oclusión estable a largo plazo, preservando la salud oclusal y articular (Figuras 10 a 13).

\section{DISCUSIÓN}

Frecuentemente los tratamientos de ortodoncia carecen de una finalización adecuada debido al desgaste que presentan los órganos dentales, y los órganos dentales que comúnmente encontramos traumatizados son los involucrados en la guía anterior, su relevancia primaria es ayudar a prevenir interferencias posteriores en movimientos excéntricos permitiendo a los cóndilos moverse. ${ }^{3,4}$

La ameloplastia positiva siempre ha sido fundamental como parte de la finalización en un tratamiento de ortodoncia, pero los avances estéticos y funcionales que existen en la actualidad han permitido evolucionar y facilitar la finalización de los casos, ya que éstos permiten alcanzar objetivos en los cuales la ortodoncia estaba limitada. ${ }^{5}$

En pacientes jóvenes, el uso de restauraciones de composite en segmento anterior es una excelente alternativa debido a que probablemente es el tratamiento más conservador.
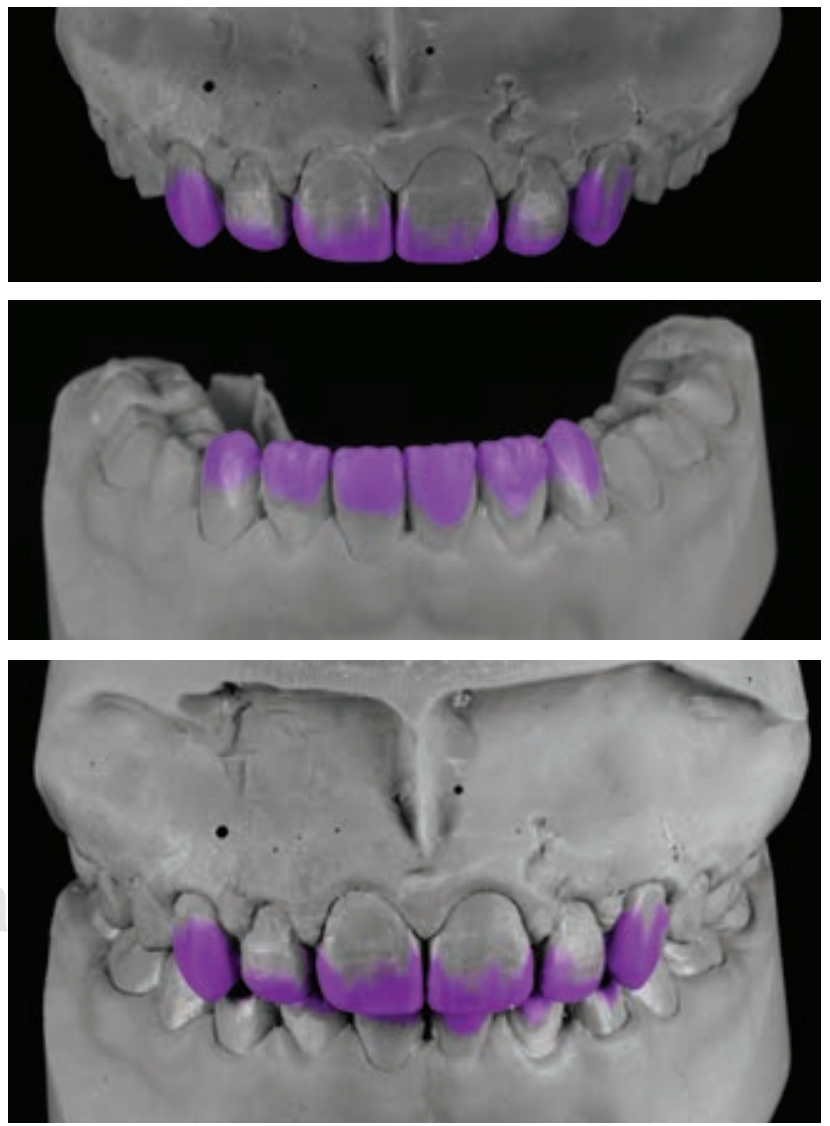

Figura 10: Montajes para el análisis de guías funcionales. 

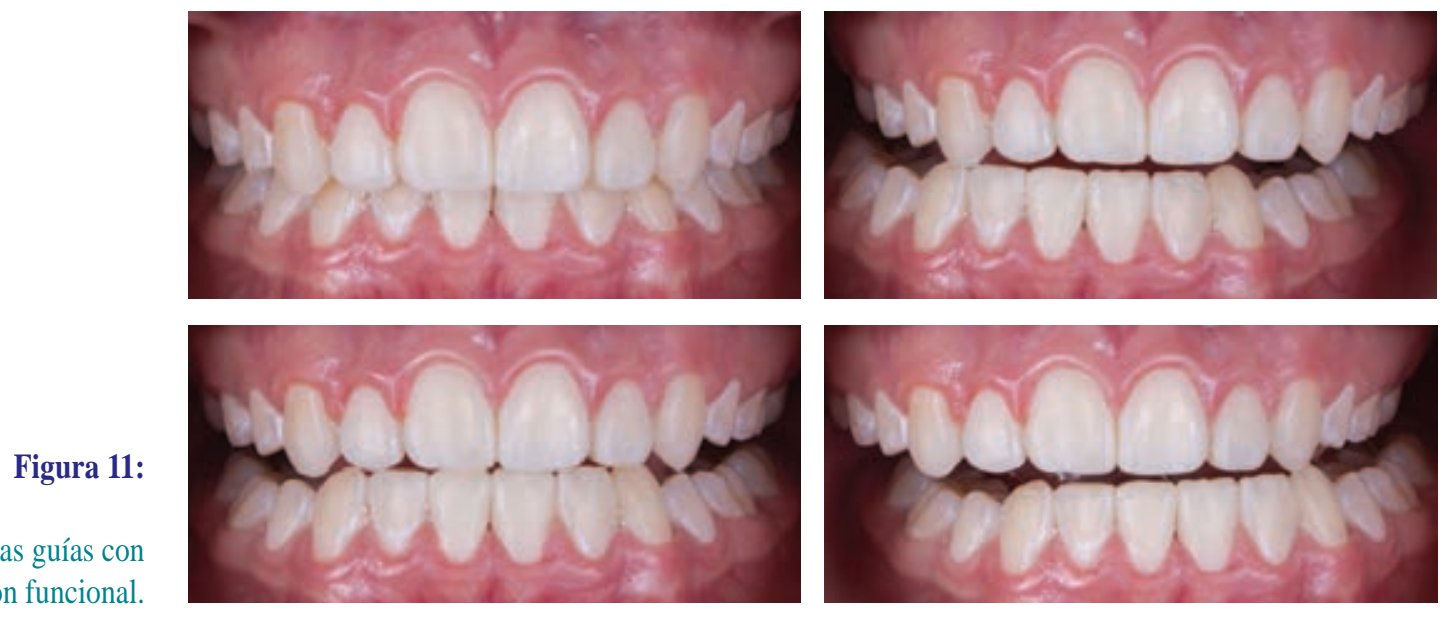

Presentación de las guías con una oclusión funcional.
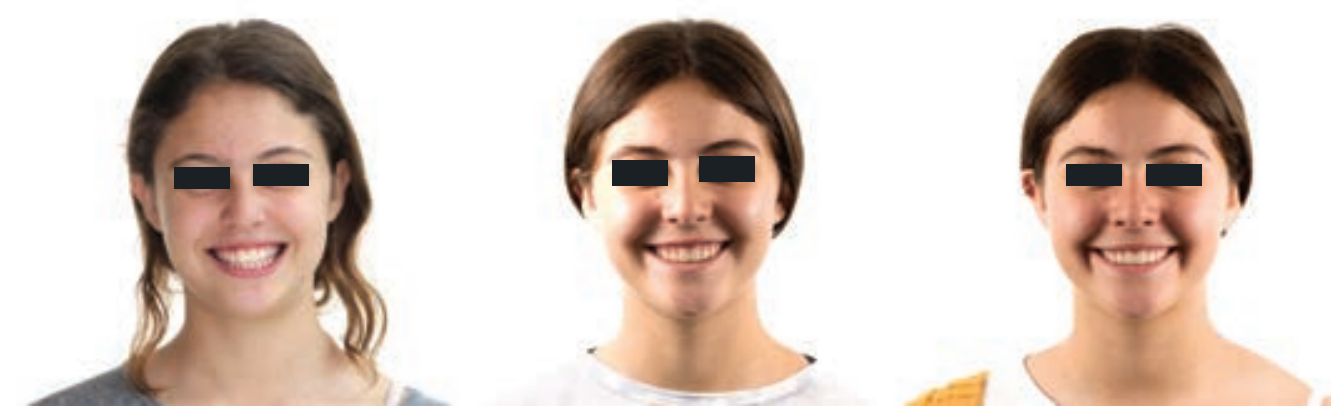

Figura 12: Secuencia fotográfica durante el tratamiento.
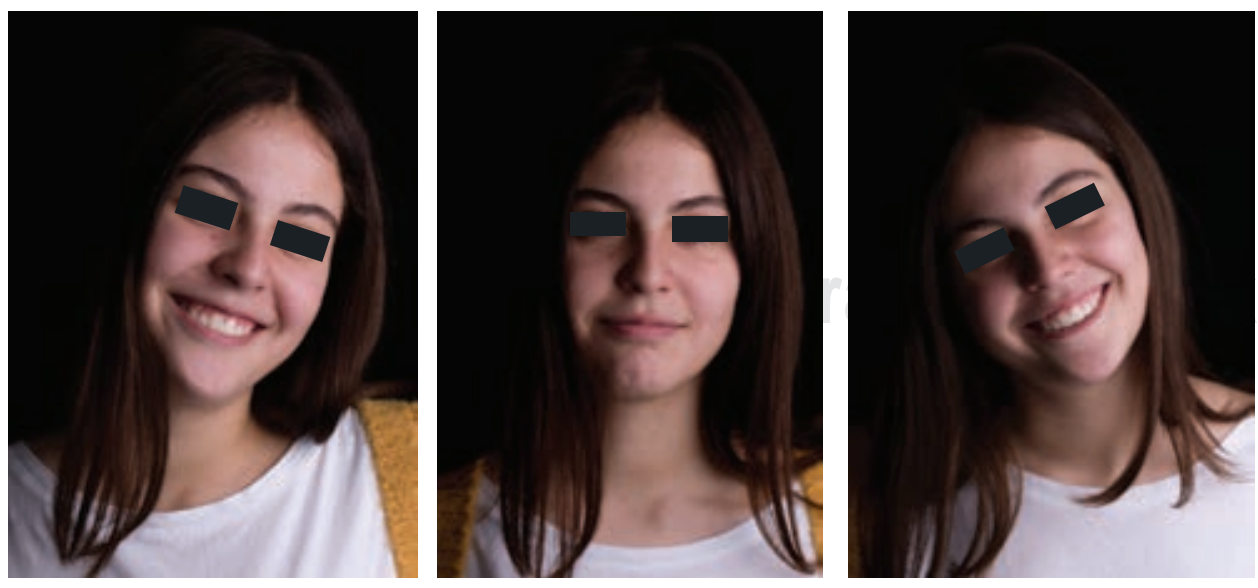

Figura 13:

Presentación final de la armonía en el caso terminado. 
La confección de estas restauraciones mediante una planificación en relación céntrica que determine la correcta forma, no sólo brinda una ventaja estética, sino funcional y estabilidad oclusal y articular a largo plazo con una oclusión mutuamente protegida. ${ }^{2}$

\section{CONCLUSIONES}

Devolver la función, forma y estética bajo los conceptos biofuncionales debe ser una prioridad en la finalización del tratamiento de ortodoncia para considerarse completo, y debe hacerse en relación céntrica para garantizar su estabilidad a largo plazo.

Los pacientes deben ser conscientes de que al realizar la reconstrucción del segmento anterior deben mantener un control anual que incluye el pulido y sellado de las restauraciones que se fabricaron con resina o realizar el recambio por un material más duradero como carillas de porcelana.

\section{BIBLIOGRAFÍA}

1. Lee RL. Anterior guidance. In: Rufenacht CR. Fundamentals of esthetics. Chicago IL: Quintessence; 1990.

2. Lee R. Chapter 5: Esthetics and it's relationship to function. In: Rufenacht CR. Fundamentals of esthetics. Chicago IL: Quintessence; 1990. pp. 1-66.

3. Okeson JP. Oclusión y afecciones temporomandibulares. 4th ed. España: Editorial Harcourt; 1998.

4. Lee RL. Jaw movements engraved in solid plastic for articular controls. I. Recording apparatus. J Prosthet Dent. 1969;22(2):209-224.

5. Solnit A, Curnutte DC. Occlusal correction: principles and practice. Chicago IL: Quintessence; 1988. pp. 87, 97.

\section{Correspondencia:}

\section{Francisco Espinosa Albo}

Blvd. Oscar Flores Núm. 809,

Col. Constitución, 32880, Ojinaga, Chihuahua.

Tel: 6142859525

E-mail: franespinosaalbo@gmail.com 